\title{
EDITORIAL
}

\section{Leksell Gamma Knife Society and radiosurgery: a legacy and a vision for the future}

\author{
Caroline Chung, MD, MS, FRCPC, CIP, ${ }^{1}$ Dheerendra Prasad, MD, MCh, FACRO, ${ }^{2}$ \\ Michael Torrens, MBBS, MPhil, ChM, FRCS, ${ }^{3}$ lan Paddick, MS, ${ }^{4}$ Patrick Hanssens, MD, ${ }^{5}$ \\ Douglas Kondziolka, MD, ${ }^{6}$ and David A. Jaffray, $\mathrm{PhD}^{7}$
}

\begin{abstract}
${ }^{1}$ Division of Radiation Oncology, The University of Texas MD Anderson Cancer Center, Houston, Texas; ${ }^{2}$ Department of Radiation Oncology and Neurosurgery, Roswell Park Comprehensive Cancer Center, Buffalo, New York; ${ }^{3}$ Department of Neurosurgery, Hygeia Hospital, Marousi, Greece; ${ }^{4}$ Gamma Knife, Cromwell Hospital, London, United Kingdom; ${ }^{5}$ Department of Radiation Oncology, Elisabeth-Tweesteden Hospital, Tilburg, The Netherlands; ' $D$ Department of Neurosurgery, NYU Langone Medical Center, New York, New York; and 'Departments of Radiation Oncology and Medical Biophysics, University of Toronto, and Department of Medical Physics, Radiation Medicine Program, Princess Margaret Cancer Centre/University Health Network, Toronto, Canada
\end{abstract}

$\mathrm{M}$ ANY professional societies have been founded by and continue to support a single discipline. Within the field of medicine, the reality of clinical patient care has increasingly evolved toward multidisciplinary communication, collaboration, and care coordination; however, many societies continue to have meetings that largely focus on a single discipline.

Nearly 30 years ago, Dr. Dan Leksell recognized that, although the roots of radiosurgery were born out of neurosurgery, there was a growing interdependence with medical physics and radiation oncology. To foster the collaborative growth of a multidisciplinary community, he had the forward-thinking vision to establish the Leksell Gamma Knife Society (LGKS) to provide a forum for multidisciplinary exchange of ideas and information across disciplines: physicians, medical physicists, and basic scientists, with the purpose of improving patient outcomes. This effort was largely underwritten with support from Elekta AB (Stockholm, Sweden), a company began by Professor Lars Leksell. Their uncompromising support led not only to the creation of devices and technologies but also to a medical field. Initial meetings took place beginning in 1989. Charlottesville (US), Pittsburgh (US), Bath (UK), Buenos Aires (Argentina), and Aronsborg (Sweden) were some of the initial conference locations. Biannual meetings were soon attended by hundreds of participants as the meeting sites rotated among North America, Europe, and Asia-Australia.

Dan Leksell shared that "at the very beginning, stereotactic radiosurgery (SRS) was the Gamma Knife but we came together around the methodology of SRS. The goal of the LGKS was to share information openly across the SRS community." Aligned with this goal, the official mission of the LGKS is "to further define and expand the role of Gamma Knife Surgery in the treatment of intracranial disorders. The development of best practices in order to improve patient outcomes is an important objective of the Society." Interdisciplinary camaraderie helped to diffuse the typical rivalries that different specialties often have.

To serve this mission, the LGKS has helped initiate a number of collaborative educational activities and research projects over its 30-year history. The recurring course for functional radiosurgery, entitled the "Noble Art of Lesioning," provides training for functional Gamma Knife radiosurgery for conditions such as tremor, pain, and obsessive-compulsive disorder. The Society supports numerous fellowships at host institutions where young neurosurgeons and oncologists are able to spend 3 months at well-regarded radiosurgery centers in the US and Europe. Multiple clinical and technical research projects have been initiated by the Society and its membership over the years and have led to multicenter studies. The LGKS has supported recurring scientific meetings of the Society since 1989 , with the initial meeting proceedings between 1989 and 1999 published in Stereotactic and Functional Neurosurgery and since 2000 published in the Journal of Neurosurgery on a biennial basis. These meetings are known for their open dialogue and debate among the multidisciplinary participants.

As a reflection of these efforts, the LGKS has grown into a multinational and multidisciplinary community that embraces open dialogue around advances in technology 
and science but also tackles the challenging discussions on variations in practice and individual roles across a multidisciplinary team. The rapid growth in this global community is demonstrated in the formation of 4 additional regional societies, the North American Gamma Knife Consortium (NAGKC) and the Japanese (JLGKS), Asian (ALGKS), and European (EGKS) Gamma Knife Societies, which have worked together to publish numerous larger-scale clinical research studies on radiosurgery indications and outcomes that have served as major benchmarks in the field. The NAGKC became the International Gamma Knife Research Foundation and now more recently, the International Radiosurgery Research Foundation. Other technology-based groups followed suit, and groups such as the CyberKnife Society, the Radiosurgery Society, and Novalis Circle have subsequently formed.

Through the open dialogue across regions and across disciplines, it was realized that variability in treatment planning processes, dosimetric reporting, and assessment of treatment response and toxicity data has hindered meaningful systematic data analyses or cross-comparison of results across studies in the field of SRS. The engagement and open communication within the multidisciplinary membership led the LGKS to take a major forwardthinking step in creating a multidisciplinary standardization committee in 2012 to establish recommendations for uniform terminology and standardized dose reporting for radiosurgery. The goal of this standardization effort is to enable the entire radiosurgery community-neurosurgery, radiation oncology, medical physics, and others-to communicate clearly and to collaborate effectively, continuing in its pursuit of improving patient care. Standardized measurement promises to enhance our research and ability to interpret the impact of treatment on outcomes across heterogeneous practices, thereby helping us deliver personalized treatments. Validation and demonstration of the value of standardized nomenclature and measurement moving forward is dependent on the adoption of these standardized practices.

In 2014, the LGKS standardization committee published its white paper entitled "Standardization of terminology in stereotactic radiosurgery: report from the Standardization Committee of the International Leksell Gamma Knife Society: special topic" in the Journal of Neurosurgery. ${ }^{10}$ Drawing on the strengths of a collaborative multidisciplinary community, the intent of this initial white paper was to align radiosurgery dose reporting with the International Commission on Radiation Units and Measurements (ICRU) frameworks. ${ }^{2}$ The ICRU has established standards for radiation units and measurement for nearly 90 years, and radiation oncology practices have largely adopted the use of the ICRU standardized nomenclature and radiotherapy dose reporting practices for many years. This was followed by a second report, "Assessment of organs-at-risk contouring practices in radiosurgery institutions around the world-the first initiative of the OAR Standardization Working Group," published in Radiotherapy and Oncology in 2016, which provided a critical and sobering evaluation of the variabilities across global radiosurgery practices. ${ }^{8}$ The importance of a coordinated multidisciplinary standardization effort has been acknowledged through endorsement and partnership with the International Society of Stereotactic Radiosurgery (ISRS) in 2014, in a unified effort to standardize the reporting of all radiosurgery treatments as well as its outcomes through collaboration on a globally standardized terminology, OAR definition, dose reporting, and outcome reporting practice. Globally, the effort to provide guidance on standardization and reporting is now maturing, with recent publication of ICRU Report $91^{9}$ and by AAPM Task Group $263^{3}$ representing more in-depth efforts that highlight the multidisciplinary engagement across medical physics and radiation oncology, reflecting the earlier efforts initiated by the LGKS. ICRU Report 91, "Prescribing, recording, and reporting of stereotactic treatments with small photon beams," was published in 2017. ${ }^{9}$ Shortly after this publication, in January 2018, AAPM Task Group 263 published its report entitled "Standardizing Nomenclatures in Radiation Oncology." 3 These efforts across medical physics and radiation oncology follow and complement the earlier efforts initiated by the LGKS.

Looking back, the LGKS and the radiosurgery community have led the forefront of not only open multidisciplinary discussion but also education and research collaboration with the focus of improving patient care. Back in September 2010, initial discussions around establishing an LGK registry for patients treated with SRS on the Gamma Knife systems took place. As with many concepts that are well ahead of their time, this registry has not fully materialized, but it has inspired the inception of subsequent radiosurgery-related registries, including the ASTRO/ AANS registry. Overall, the scientific community at-large is only now learning to appreciate the full potential of open science and collaborative learning, and failed efforts are critical contributors to this emerging appreciation. Working as multidisciplinary teams for clinical care, research, and education are key steps toward meaningful aggregation of data and the development of clinical insights.

As we look forward in this era of big data, open science, and a global medical community, we need to acknowledge that an essential yet very challenging step is the implementation of our established standards. Implementation, the process of putting a plan into effect, requires significant commitment and collaborative effort across the continuum of clinical care, research, and educational activities. The global SRS community is faced with the responsibility and challenge of working to adopt standardized and structured data collection across disciplines and practices and across time. This requires expansion of the currently acknowledged members of the multidisciplinary team to include data scientists and the industry partners who provide the hardware and software solutions. The standards need to be directly supported in the software tools (planning and information systems) we use for clinical practice and should facilitate standardization through integrated nomenclature support, workflow automation, and tools that readily enable reporting to international data repositories. In the same manner that standardized Brain Tumor Imaging Protocols ${ }^{1}$ have been implemented as a requirement for future brain tumor clinical trials by a collaborative effort across the Food and Drug Administration, National Cancer Institute, and major stakeholders 
of brain tumor clinical trials, we need a concerted effort to require future radiosurgery clinical trial protocols to use the established SRS dose, volume, and OAR reporting standards. The scientific community needs to ensure our published literature is also following these standards. Just as the REMARK standard is being adopted for biomarker publication by major journals in oncology, ${ }^{4-7}$ we need similar reinforcement of the dose, volume, and OAR reporting standards that have emerged from the efforts of the ISRS and LGKS community in the publication review processes of our major journals. ${ }^{10}$ Last but not by any means least, our educational programs need to be validated for their conformance to teaching these standards to ensure that future generations are able to accelerate collaborative evidence development and communicate effectively across disciplines, institutions, and treatment modalities in order to practice at and push the limits of the technology and continually improve clinical care.

At this year's LGKS meeting in Dubai, it was announced that the LGKS biennial meeting would be forever folded into the ISRS meeting. The announcement was bittersweet: while it was the end of a series of engaging multidisciplinary events spanning 30 years, its legacy is the many progressive concepts and successes that should inspire and encourage the larger radiosurgery community to continue open dialogue and strive for global multidisciplinary collaboration to advance patient care.

https://thejns.org/doi/abs/10.3171/2018.7.GKS181992

\section{Acknowledgments}

We would like to thank Danny Leksell for his leadership and vision over the years and for his support of this editorial.

\section{References}

1. Ellingson BM, Bendszus M, Boxerman J, Barboriak D, Erickson BJ, Smits M, et al: Consensus recommendations for a standardized Brain Tumor Imaging Protocol in clinical trials. Neuro Oncol 17:1188-1198, 2015

2. Landberg T, Chavaudra J, Dobbs J, Gerard JP, Hanks G, Horiot JC, et al: Prescribing, Recording and Reporting Photon Beam Therapy (Supplement to ICRU Report 50). Bethesda, MD: International Commission on Radiation Units and Measurements, 1999 (https://academic.oup.com/jicru/ article/os32/1/NP/2924047) [Accessed July 25, 2018]
3. Mayo CS, Moran JM, Bosch WR, Xiao Y, McNutt T, Popple $\mathrm{RA}$, et al: Standardizing Nomenclatures in Radiation Oncology. Alexandria, VA: American Association of Physicists in Medicine, 2018 (https://www.aapm.org/pubs/ reports/RPT_263.pdf) [Accessed July 25, 2018]

4. McShane LM, Altman DG, Sauerbrei W, Taube SE, Gion M, Clark GM, et al: Reporting recommendations for tumor marker prognostic studies (REMARK). J Natl Cancer Inst 97:1180-1184, 2005

5. McShane LM, Altman DG, Sauerbrei W, Taube SE, Gion M, Clark GM, et al: REporting recommendations for tumor MARKer prognostic studies (REMARK). Nat Clin Pract Oncol 2:416-422, 2005

6. McShane LM, Altman DG, Sauerbrei W, Taube SE, Gion M, Clark GM, et al: REporting recommendations for tumour MARKer prognostic studies (REMARK). Eur J Cancer 41:1690-1696, 2005

7. McShane LM, Altman DG, Sauerbrei W, Taube SE, Gion M, Clark GM, et al: REporting recommendations for tumour MARKer prognostic studies (REMARK). Br J Cancer 93:387-391, 2005

8. Sandstrom H, Chung C, Jokura H, Torrens M, Jaffray D, Toma-Dasu I: Assessment of organs-at-risk contouring practices in radiosurgery institutions around the world-The first initiative of the OAR Standardization Working Group. Radiother Oncol 121:180-186, 2016

9. Seuntjens J, Lartigau E, Cora S, Ding GX, Goetsch S, Nuyttens J, et al: Prescribing, recording, and reporting of stereotactic treatments with small photon beams. Report 91. J ICRU 14:1-160, 2014

10. Torrens M, Chung C, Chung HT, Hanssens P, Jaffray D, Kemeny A, et al: Standardization of terminology in stereotactic radiosurgery: report from the Standardization Committee of the International Leksell Gamma Knife Society: special topic. J Neurosurg 121 Suppl:2-15, 2014

\section{Disclosures}

Caroline Chung, research funding (Elekta); Dheerendra Prasad, consultant (Elekta); Ian Paddick, consultant (Elekta); Doug Kondziolka, research funding (Brainlab); David Jaffray, patent holder (William Beaumont Hospital/Elekta, University Health Network/Elekta).

\section{Correspondence}

Caroline Chung: cchung3@mdanderson.org.

INCLUDE WHEN CITING

DOI: 10.3171/2018.7.GKS181992. 